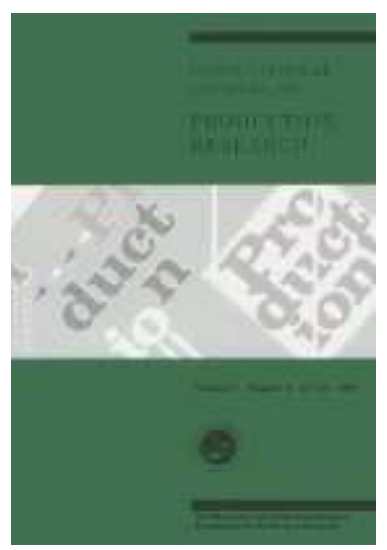

\title{
Dynamic Re-order Point Inventory Control with Lead-Time Uncertainty: Analysis and Empirical Investigation
}

\begin{tabular}{|c|c|}
\hline Journal: & International Journal of Production Research \\
\hline Manuscript ID: & TPRS-2007-IJPR-0176.R1 \\
\hline Manuscript Type: & Original Manuscript \\
\hline $\begin{array}{r}\text { Date Submitted by the } \\
\text { Author: }\end{array}$ & 23-Jul-2007 \\
\hline Complete List of Authors: & $\begin{array}{l}\text { Babai, Mohamed; University of Salford, Salford Business School } \\
\text { Syntetos, Aris; University of Salford, Salford Business School } \\
\text { Dallery, Yves; Ecole Centrale Paris, Laboratoire Genie Industriel } \\
\text { Nikolopoulos, Kostantinos; University of Manchester, Manchester } \\
\text { Business School }\end{array}$ \\
\hline Keywords: & $\begin{array}{l}\text { INVENTORY CONTROL, FORECASTING, MANUFACTURING } \\
\text { MANAGEMENT, INVENTORY MANAGEMENT, PRODUCTION } \\
\text { ECONOMICS }\end{array}$ \\
\hline Keywords (user): & Stock Control, Demand Forecasting \\
\hline
\end{tabular}

\section{ScholarONE" \\ Manuscript Central}




\title{
Dynamic Re-order Point Inventory Control with Lead-Time Uncertainty: Analysis and Empirical Investigation
}

\author{
M. Zied Babaï ${ }^{* \#}$, Aris A. Syntetos*, Yves Dallery ${ }^{* *}$ and Konstantinos Nikolopoulos ${ }^{* * *}$ \\ *Centre for Operational Research and Applied Statistics (CORAS), Salford Business School, \\ University of Salford, Maxwell Building, Greater Manchester M5 4WT, UK. \\ m.z.babai@salford.ac.uk, a.syntetos@ salford.ac.uk \\ ***aboratoire Génie Industriel, Ecole Centrale Paris, Grande Voie des Vignes, 92290 \\ Chatenay Malabry, France \\ dallery@lgi.ecp.fr \\ *** Manchester Business School, University of Manchester, Booth Street West, Manchester \\ M15 6PB, UK. \\ kostas.nikolopoulos@mbs.ac.uk \\ \# Corresponding author
}

\begin{abstract}
A new forecast-based dynamic inventory control approach is discussed in this paper. In this approach, forecasts and forecast uncertainties are assumed to be exogenous data known in advance at each period over a fixed horizon. The control parameters are derived by using a sequential procedure. The merits of this approach as compared to the classical one are presented. We focus on a single-stage and single-item inventory system with non-stationary demand and lead-time uncertainty. A dynamic re-order point control policy is analysed based on the new approach and its parameters are determined for a given target cycle service level (CSL). The performance of this policy is assessed by means of empirical experimentation on a large demand data set from the pharmaceutical industry. The empirical results demonstrate the benefits arising from using such a policy and allow insights to be gained into other pertinent managerial issues.
\end{abstract}

Keywords: Stock Control; Forecasting; Lead-time Demand; Simulation; Empirical Analysis

\section{Acknowledgements}

The research conducted by the first two authors (M.Z. Babai and A.A. Syntetos) was supported by the Engineering and Physical Sciences Research Council (EPSRC, UK) grant EP/D062942/1. (More information on this project may be found at: http://www.mams.salford.ac.uk/CORAS/Projects/Forecasting/) 


\section{Introduction}

The efficient management of inventories is an important concern of all companies that deal with physical stock at any level of a given supply chain. Inventory control is viewed as an important task since it is the lever enabling significant cost reductions and/or higher customer service levels. This task becomes more complex in the context of highly variable customer demand and uncertain replenishment lead times.

Many academic investigations have been performed since the 1930's in order to develop or to analyze new inventory control policies that can be implemented in real inventory management systems. In the context of highly variable demand, the standard "static" control policies such as the static re-order point or the static orderup-to-level policy are often inappropriate, since they are based on the stationary demand assumption. It is essential to develop dynamic inventory control policies considering non-stationary demand, since such policies account better for the variable patterns encountered in practical situations.

In many real-world cases, companies are still using static policies to compute the ordering quantities and safety stocks for non-stationary independent demands. These quantities are often derived from histories of demand, and are rarely reviewed. This is the case even when demand forecasts are available. Hence, dynamic inventory control systems aiming to tackle this issue must be developed in order to help practitioners in their decision making process.

Some academic research work has dealt with dynamic inventory control policies under non-stationary demand (with or without the use of forecasts). However, the methods developed in this work are either not easy to implement by practitioners (i.e. computationally intensive and/or not intuitively appealing, (e.g. Scarf, 1959; Karlin, 1960; Hadley and Whitin, 1963), or they are based on restrictive assumptions which make their implementation strongly dependent upon specific underlying demand patterns and forecasting methods (e.g. Heath and Jackson, 1994; Graves, 1999; Chen et al., 2000; Strijbosch et al., 2000; Syntetos and Boylan, 2006).

Furthermore, in addition to demand uncertainty, the uncertainty associated with replenishment lead-times significantly affects the performance of the inventory systems. Most relevant literature relies on the assumption of constant lead-times and/or stochastic lead-times, but also stationary demand. 
In this paper, we discuss a new approach to forecast-based inventory control. Our aim is to develop a simple dynamic inventory control policy that can be easily implemented in practice, under a non-stationary demand pattern estimated by any forecasting procedure and under stochastic lead times. We assume that the forecasts, as well as the probability distributions associated with the forecast uncertainty and the lead-time, are exogenous data known in advance at each period over a fixed horizon. Our approach avoids the explicit reliance on specific forecasting methods. It allows the consideration of forecasts derived from ad-hoc methodologies, or from forecasts generated by specialised statistical forecasting software (in which case the exact updating algorithms are rarely known). Moreover, our approach enables experimentation with judgementally-adjusted or qualitative forecasts generated from relevant management and/or marketing intelligence.

Babaï and Dallery (2007) conducted some preliminary work on the above dynamic inventory control approach. In this paper we extend their analysis by considering lead- time uncertainty and by assessing the performance of a stock control policy (the re-order point one), by means of empirical experimentation on a large demand data set from the pharmaceutical industry. Two models of forecast uncertainty are considered: an absolute model and a relative model. Experimentation with the latter model constitutes a contribution by itself, because not much work has been done in this area. The remainder of this paper is organised as follows: in Section 2 we overview first the stock control literature that deals with stochastic lead-times, and then, the dynamic approaches to inventory control, contrasting them with our own approach. In Section 3 we discuss the assumptions on which our approach is developed, along with the standard dynamic re-order point policy upon which we build our work. In Section 4 we analyse the dynamic policy that considers the impact of lead-time uncertainty. In Section 5 we present the experimental structure of our empirical investigation. In Section 6 the empirical results are analysed, and in Section 7 we give our conclusions, along with some natural extensions for further work. 


\section{Research Background}

In this section we provide an overview of the stock control literature that deals with stochastic lead-times. Then we focus on dynamic inventory control policies, and discuss the relevant issues to give a background for the development of our approach.

\subsection{Inventory systems with stochastic lead-times}

Early investigations demonstrated the economic implications of lead-time uncertainty and showed the importance of ameliorating its effects, regardless of the procedures used to compensate for demand uncertainty (e.g. Whybark and Williams, 1976; Speh and Wagenheim, 1978; Nevison and Burstein, 1984).

A considerable amount of academic work has also been devoted to the analysis of periodic inventory control systems with deterministic demands and stochastic lead times (e.g. Kaplan, 1970; Tang and Grubbström, 2004). Erhardt (1984) took forward the work conducted by Kaplan (op. cit.) and studied the conditions of optimality related to the periodic $(s, S)$ policy, where $s$ is the re-order point and $S$ is the order-upto-level, for both finite and infinite planning horizons. Johnson and Montgomery (1974) and Zipkin (2000) discuss periodic inventory control systems operating under both stochastic demands and lead-times. However, the focus has been on static inventory control policies that rely on the stationary demand assumption.

Eppen and Martin's (1988) work is very relevant to our own, given their consideration of non-stationary demand and lead-time uncertainty for the purpose of computing safety stocks (under a fill rate service level constraint).

\subsection{Dynamic Inventory Control Policies}

There are two main streams of literature dealing with dynamic inventory control policies for non-stationary demand. The first is based on the earlier work by Scarf (1959), Karlin (1960) and Hadley and Whitin (1963). This literature investigates optimal inventory control policies aimed at minimising total inventory costs, including backlog penalties. It considers non-stationary stochastic demands over periods of an infinite horizon. It shows that optimal control is obtained in the form of dynamic $(s, S)$ policies. Nevertheless, the relevant parameters cannot be determined easily. That is to say, the proposed solutions are difficult to understand from a 
practitioner perspective and difficult to implement (computationally intensive/demanding). Moreover, the optimal policies have been based on minimising the total inventory cost function under restrictive assumptions. Systems often operate under a service level constraint, and should this be the case, a different approach is needed.

A body of literature has emerged more recently which studies forecast-based dynamic inventory control policies. Interested readers are referred to Heath and Jackson (1994), Lee et al. (1997), Graves (1999) and Chen et al. (2000). These authors consider auto-correlated non-stationary demand (often for fast moving items), for which forecasts are obtained using statistical forecasting methods. This work goes on to develop dynamic order-up-to-level policies where control parameters are computed under service level constraints. Nevertheless, there is an implicit exclusion of forecasts derived from ad-hoc methodologies and/or in a qualitative manner (judgemental forecasts). In addition, all the above work has been generated on restrictive assumptions, and does not take into account important practical concerns such as lead-time uncertainty.

In this paper we propose a simple approach that addresses the practical needs of inventory managers in the context of non-stationary demand by considering lead-time uncertainty. The advance demand information is given by forecasts and the objective is to satisfy a target cycle service level. The new forecast-based inventory control approach addresses, at least partly, some of the drawbacks of the classical approaches cited above. In fact, we assume that forecasts and the probability distributions of the forecast uncertainties are exogenous data known in advance over a fixed horizon. In order to derive the parameters of a given control policy, say the re-order point policy, we use a sequential procedure under a service level constraint. This means that the value of the ordering quantity is computed ignoring the impact on the re-order point. In order to compute the re-order point, a target cycle service level (CSL) is set which represents the probability of no stock-out during a cycle (a cycle being the time period between two successive orders). The framework of our approach is summarised in Figure 1.

$\ll<$ Please insert Figure 1 about here $\gg>$ 
This approach has three merits compared to the approaches cited above: i) it is generic, because the resulting control policy does not depend upon any forecasting procedure/parameters; ii) it takes lead-time uncertainty into account; and iii) it is less computationally intensive compared with other dynamic approaches proposed in the literature - such approaches deliver optimal results under very specific assumptions. Moreover, if forecasts are obtained using only statistical forecasting methods, and the probability distributions of forecast uncertainties are obtained using the forecast errors given by the underlying forecasting model, the classical approach of forecast-based inventory control collapses to our approach.

\section{System Description}

\subsection{Demand Forecasts and Uncertainty}

We analyse a single-stage and single-item inventory control system under nonstationary demand, information about which is provided through forecasting. The system is not capacitated and the inventory replenishment requires a lead-time, as presented in Figure 2.

\section{$\ll<$ Please insert Figure 2 about here $\gg>$}

Two key elements must be available as exogenous data just before the beginning of the forecast horizon in order to estimate future requirements and to set the control parameters, as shown in Figure 3: i) the point forecasts over all periods in the forecast horizon, and ii) a Probability Distribution Function $(P D F)$ for the forecast uncertainty.

\section{$\ll<$ Please insert Figure 3 about here $\gg>$}

For the purposes of this research, the uncertainty associated with the provided forecasts is referred to as the 'forecast uncertainty', whereas other authors often refer to it as the 'forecast error'. We adopt this terminology since we assume that the relevant information is an exogenous indicator of the uncertainty involved in the provided demand forecasts, and that it is not only the error resulting from a statistical forecasting method. 
Generally, the random forecast uncertainty can be absolute (or additive), relative (or multiplicative), or mixed. The forecast uncertainty is absolute if it is independent of the forecasts. It is relative if it is proportional to the forecasts, and it is mixed if it consists of both an absolute and a relative component. In this paper, we assume that the forecast uncertainty may be either absolute or relative. Hence, for a period $k$, if $F_{k}$ denotes the forecast produced for the demand in that period and $F U$ denotes the random forecast uncertainty associated with that estimate, a representation of the actual demand, denoted by $D_{k}$, is given by:

$D_{k}= \begin{cases}F_{k}+F U & \text { If the forecastuncertaint is absolute } \\ (1+F U) F_{k} & \text { If the forecastuncertainty is relative }\end{cases}$

At this point it is important to mention that if the forecast uncertainty is relative, the $P D F$ is given in percentages (i.e. the mean and the standard deviation of the forecast uncertainty as well as the realisations are expressed in percentages).

To the best of our knowledge, most of the literature that deals with forecast-based inventory control policies was developed by considering absolute forecast uncertainties. However, in many cases encountered in practice, the forecast uncertainty for some SKUs may be better represented by a relative or a mixed model.

In inventory control, the safety stock covers the cumulative forecast uncertainty over the protection interval, which in periodic review systems is equal to the lead time plus one review period. Therefore, the $P D F$ of the cumulative forecast uncertainty over the protection interval is needed. The literature suggests that the common way to do this is by aggregating the $P D F$ s of the forecast uncertainties associated with each period over the protection interval (cf. Armstrong, 2001). For example, assume that at the end of period $k$, the PDFs of the forecast uncertainty are known for all periods $i$ of a protection interval $R$ (i.e. $i=k+1, k+2, . ., k+R-1$ ), that is respectively $P D F(k+1)$, $P D F(k+2), \ldots, P D F(k+R-1)$. In this case, the forecaster usually creates at the end of period $k$ an Aggregate $P D F$ (say $A P D F(k)$ ) with mean and variance given by:

$$
\begin{aligned}
& \mu_{A P D F(k)}=\mu_{P D F(k+1)}+\mu_{P D F(k+2)}+. .+\mu_{P D F(k+R-1)} \text { and } \\
& \sigma_{A P D F(k)}^{2}=\sigma_{P D F(k+1)}^{2}+\sigma_{P D F(k+2)}^{2}+. .+\sigma_{P D F(k+R-1)}^{2} \text { respectively }{ }^{1} .
\end{aligned}
$$

\footnotetext{
${ }^{1}$ Those expressions of the mean and the variance are available only in the case of an absolute model of the forecast uncertainty.
} 
Although common in practice, however, this approach is not intuitively appealing, as it does not allow the consideration of autocorrelations of forecast uncertainties over the protection interval. This could only be achieved via a true Cumulative $P D F$, and that is what we suggest should be adopted. In Appendix A we provide a simple method for determining the parameters of the Cumulative PDF of the forecast uncertainty. Empirical results showing the numerical supremacy of this approach are presented in section 6. For the moment we conclude that a $P D F$ that could handle an interval $R$ composed of multiple periods as one 'large' period should lead to lower inventories, and is therefore regarded as a more cost-effective solution.

Let us assume now that forecasts and the $P D F$ of the cumulative forecast uncertainty over any interval $R$ (interval composed of $R$ periods) are known at each period of the horizon. For the remainder of our paper, we denote by:

$F_{k, j}$ : the forecast given at the end of period $k$ for period $j(j \geq k+1)$

$C F U_{R}$ : the random cumulative forecast uncertainty over an interval $R$

$\Phi_{C F U_{R}(.)}:$ the probability distribution function of $C F U_{R}$

$\mu_{C F U_{R}}:$ the mean of the cumulative forecast uncertainty $C F U_{R}$

$\sigma_{C F U_{R}}$ : the standard deviation of the cumulative forecast uncertainty $C F U_{R}$

$I_{k}:$ the inventory position at the end of period $k$

$L:$ the replenishment lead-time

$C S L$ : the target cycle service level

$A$ : the fixed ordering cost

$h$ : the unit holding cost

$H$ : the number of periods in the horizon of forecasts.

\subsection{The $\left(r_{k}, Q\right)$ Policy}

In this subsection, we consider the system described above for the dynamic periodic re-order point $\left(r_{k}, Q\right)$ control policy. Under this policy the system is controlled at the end of every review period. At the end of each period $k-1$, if the inventory position falls below the re-order point $r_{k}$, a quantity $Q$ is ordered. The quantity ordered is 
received after $L$ periods. The inventory level evolution in the $\left(r_{k}, Q\right)$ policy is graphically presented in Figure 4.

\section{$\ll<$ Please insert Figure 4 about here $\gg>$}

Under this policy the protection interval is equal to the sum of the replenishment leadtime $L$ plus the review period ${ }^{2}$. The single period must be added due to the discrete nature of the time review. Thus, the re-order point $r_{k}$ is equal to the cumulative forecast and the cumulative forecast uncertainty over $L+1$ periods for the specified cycle service level. The re-order point is given by:

$r_{k}= \begin{cases}\sum_{j=1}^{L+1} F_{k-1, k+j-1}+\Phi_{C F U_{L+1}}^{-1}(C S L) & \text { If the forecast uncertaint y is absolute } \\ \sum_{j=1}^{L+1} F_{k-1, k+j-1}\left[1+\Phi_{C F U_{L+1}}^{-1}(C S L)\right] & \text { If the forecast uncertaint y is relative }\end{cases}$

In the case of an absolute forecast uncertainty, the safety stock which corresponds to the term $\Phi_{C F U_{L+1}}^{-1}(C S L)$ is constant over time. In the case of a relative forecast uncertainty, the safety stock given by $\Phi_{C F U_{L+1}}^{-1}(C S L) \sum_{j=1}^{L+1} F_{k-1, k+j-1}$ is variable over time.

The ordering quantity $Q$ can be set based on the constraints and characteristics of the inventory system. It may also be computed once and for all (at the beginning of the horizon) by balancing the inventory holding costs and the ordering costs by using, for example, Wilson's formula:

$Q=\sqrt{\frac{2 A \sum_{i=1}^{H} F_{0, i}}{h H}}$

where $F_{0, i}$ denotes the forecast given at the beginning of the horizon for a period $i$.

\footnotetext{
${ }^{2}$ Correspondingly in real continuous/transaction reporting applications of re-order point policies, the requirements are calculated over the lead-time.
} 
The quantity $Q$ can also be dynamically optimized based on more complex methods such as the Wagner-Whitin method (Wagner and Whitin, 1958), or more recent alternatives (e.g. Wagelmans et al., 1992; Aggarwal and Park, 1993). Another more simple but not optimal method is the Silver-Meal heuristic (Silver, 1978).

It is easy to show that if demand is stationary and the forecasts are constant, the $\left(r_{k}, Q\right)$ policy is equivalent to the standard static $(r, Q)$ policy. Thus, the latter policy can be considered as a particular case of the former one. Furthermore, for a single stage inventory system and in the case of an absolute forecast uncertainty, the $\left(r_{k}, Q\right)$ policy is equivalent to the single stage MRP policy using a constant safety stock and a fixed order quantity $Q$ (Babai and Dallery, 2007).

\section{The $\left(r_{k}, Q\right)$ Policy with Lead-Time Uncertainty}

The system discussed in the previous section is further analysed here under the realistic assumption that the replenishment lead-time $L$ is a random variable. Our aim is to provide a simple method that can be used to compute the control parameters of the $\left(r_{k}, Q\right)$ policy under these circumstances.

In the remainder of this section we derive the parameters of the $\left(r_{k}, Q\right)$ policy when demand and lead-time uncertainties are considered simultaneously. The parameters are derived using a sequential procedure to satisfy a cycle service level.

Since we analyse a discrete time control system, we assume that the lead-time random variable $L$ is given by a discrete probability distribution with a mean and standard deviation denoted by $\mu_{L}$ and $\sigma_{L}$ respectively (i.e. $L$ takes on the values $L_{i}$, such as $\left.\sum_{i} P\left(L=L_{i}\right)=1\right)$

Using the sequential procedure described above, the ordered quantity is computed independently of the lead-time uncertainty. However, under the lead-time uncertainty, the expression of the re-order point $r_{k}$ changes.

\section{Proposition 1}

The re-order point $r_{k}$ can be computed numerically, at the end of period $k-1$, by resolving the equation: 
$\sum_{i} P\left(L=L_{i}\right) \Phi_{C F D_{k-1, L_{i}+1}}\left(r_{k}\right)=C S L$

where $C F D_{k-1, L_{i}+1}$ denotes the sum of the cumulative forecasts plus cumulative forecast uncertainty over $L_{i}+1$ periods, estimated at the end of period $k-1$ (i.e. $\left.C F D_{k-1, L_{i}+1}=C F U_{L_{i}+1}+\sum_{j=1}^{L_{i}+1} F_{k-1, k+j-1}\right)$ and $\Phi_{C F D_{k-1, L_{i}+1}}$ (.) its cumulative probability distribution.

The proof of Proposition 1 is given in Appendix B.

The parameters of the $\left(r_{k}, Q\right)$ policy are given as follows:

- The re-order point $r_{k}$ can be computed numerically by resolving the following equation (for example with a fixed-point algorithm):

$\sum_{i} P\left(L=L_{i}\right) \Phi_{C F D_{k-1, L_{i}+1}}\left(r_{k}\right)=C S L$

where $C F D_{k-1, L_{i}+1}$ denotes the sum of the cumulative forecasts plus cumulative forecast uncertainty over $L_{i}+1$ periods, estimated at the end of period $k-1$, and $\Phi_{C F D_{k-1, L_{i}+1}}($.) its cumulative probability distribution.

- The quantity to order $Q$, if it is computed, for example, by using the Wilson's formula, is given by: $Q=\sqrt{\frac{2 A \sum_{i=1}^{H} F_{0, i}}{h H}}$

\section{Empirical investigation}

In this section, we present a brief description of the demand data set available for the purposes of this research, along with the experimental structure of our empirical investigation.

\subsection{Empirical Data}

The empirical investigation is based on data relating to monthly demand forecasts for the UK branch of a major international pharmaceutical company. The company relies 
upon commercially available software to produce system forecasts per SKU for each time period (i.e. month). Final forecasts are produced at a later stage through the superimposition of qualitative judgements based on marketing intelligence given by the company forecasters.

The database consists of the individual demand histories of 829 SKUs. Distinct demand patterns are included in the sample, i.e. lumpy, intermittent and smooth demand patterns. Demand is recorded monthly and the available history covers 36 consecutive periods from January 2003 to December 2005 (three years inclusive).

System forecasts are available for all time periods and over a horizon of 36 periods (36 steps ahead forecast). The judgemental adjustment is also available, where applicable (with a sign, i.e. plus or minus). The statistical forecast provided by the software plus the judgemental adjustment give the final forecast, i.e. the one used for decision making purposes.

Not all series were considered for experimentation purposes. The following series were excluded from our empirical investigation:

- Series with missing demand data (i.e. blank cells in the spreadsheet - when that was the case there was a series of blank cells) or invalid recording of data (e.g. decimals, text, etc.)

- Intermittent demand series (i.e. series with some streaks of zeroes)

- Series consisting of a streak of zeroes followed by a streak of non-zero demands or the other way around (new SKUs or re-coded ones respectively).

It is important to note that there have been series containing some negative values (returns). We have opted for retaining the relevant information by replacing the negative values with zero (see also Syntetos et al., 2007).

The screening process resulted in 135 files being considered for our simulation purposes. All of them are fast moving products. More details on the demand characteristics across all SKUs are given in the following table:

$<<$ Please insert Table 1 about here $\gg>$ 


\subsection{Simulation Details}

We used the demand data set described above to empirically assess the performance of the dynamic $\left(r_{k}, Q\right)$ policy against that of the commonly employed static re-order point policy $(r, Q)$. In the former case the computed re-order points vary over the forecast horizon, depending on the relevant forecasts (which are available for the purposes of our research), whereas in the latter case one single re-order point is set, once and for all, to control inventories. We split the demand history available (i.e. 36 periods) into two parts. The first part, referred to as 'within-sample', is composed of $N_{l}$ periods, and is used as the history of demand required to determine the probability distributions of forecast uncertainties for the $\left(r_{k}, Q\right)$ policy, and to determine both $r$ and $Q$ in the $(r, Q)$ policy. The second part, referred to as the 'out-of-sample', is composed of $N_{2}=36-N_{1}$ periods, and it is used to evaluate performance for both policies. For example, if $N_{l}=21$, we use a within-sample of 21 periods from January 2003 to September 2004 to determine the probability distributions of forecast uncertainties. This information in conjunction with the forecasts available for the remaining $N_{2}=15$ periods is used to determine the subsequent 15 re-order points (for the dynamic policy) and to evaluate performance over that time period. Performance is evaluated by means of the achieved cycle service level and the total average inventory cost (average holding inventory cost + average ordering cost + average back-ordering cost). In the latter case we report the aggregate (across all SKUs) inventory cost (in $£$ ) per period (i.e. month).

Different scenarios of the widths of the within-sample/out-of-sample and lead-times are considered in our simulations in order to evaluate their impact on the performance of the $\left(r_{k}, Q\right)$ policy. In the case of the particular company that provided the empirical data, lead-times generally do not exceed twelve weeks (i.e. 3 months). In addition, we treat time as a discrete variable (months) and we introduce some variability on the lead-time length. Three discrete probability distributions are considered and the relevant details are summarised in the following table.

\section{$\ll<$ Please insert Table 2 about here $>>$}


Results are generated by considering two models of forecast uncertainties: the absolute and the relative (assuming normally distributed uncertainties). Moreover, four target cycle service levels are considered in our experiments: 0.8, 0.85, 0.9, 0.95. The different scenarios and parameter values considered in our simulations are summarised in Figure 5.

\section{$<<<$ Please insert Figure 5 about here $\gg>$}

In order to compute the $(r, Q)$ parameters: if $\mu_{D}$ and $\sigma_{D}$ denote the mean and the standard deviation of the demand respectively and if $\mu_{L}$ and $\sigma_{L}$ denote the mean and the standard deviation of the lead-time respectively, then approximations of the parameters of the discrete time $(r, Q)$ policy, derived from the sequential procedure, are as follows:

$Q=\sqrt{\frac{2 A \mu_{D}}{h}}$ and $r=\mu_{D}\left(\mu_{L}+1\right)+\Phi^{-1}(C S L) \sqrt{\left(\mu_{L}+1\right) \sigma_{D}^{2}+\sigma_{L}^{2} \mu_{D}^{2}}$

For initialisation purposes, we assume that the initial stock in both the $(r, Q)$ policy and the $\left(r_{k}, Q\right)$ policy (stock at the end of the within-sample period) is given by:

Initial stock $=r=\mu_{D}\left(\mu_{L}+1\right)+\Phi^{-1}(C S L) \sqrt{\left(\mu_{L}+1\right) \sigma_{D}^{2}+\sigma_{L}^{2} \mu_{D}^{2}}$

The unit holding cost used in our experiments is $h=0.1 £ /$ unit/period whereas the back-ordering cost $b=1 £ /$ unit/period, i.e. $h / b=10 \%$, an assumption which covers a wide range of real-world scenarios. The ordering cost is $A=200 £$ /order. Since the results in each scenario are dependent on the random realisation of lead times, many replications need to be performed in order to ensure the 'stability' of the results given by simulation. The results reported in the following section have been obtained after 5 replications of each scenario.

\section{Empirical Results}

The empirical results for the case of the absolute forecast uncertainty are summarised in the following tables for the various within-sample/out-of-sample combinations. 
$<<$ Please insert Table 3 about here $>>$

$<<<$ Please insert Table 4 about here $\gg>$

$\ll<$ Please insert Table 5 about here $\gg>$

The results indicate that as the lead-time increases, the achieved CSL decreases and the inventory cost increases. This is true for both policies considered in our experiment and is in accordance with theoretical expectations. That is to say, higher lead-times (coupled with their stochastic nature, as simulated for the purposes of our experiment) imply higher uncertainty that is reflected in increased backlog and correspondingly increased inventory costs. Moreover, the results indicate that as the target CSL increases, the achieved CSL increases as well (at the expense of a higher inventory cost). However, it becomes more difficult to meet the actual target. For target CSL $=0.8,0.85$ both policies offer CSL over and above the targets. When the $\mathrm{CSL}=0.90$, the static policy continues to exceed the target; the same is true for the $\left(r_{k}, Q\right)$ policy when its performance is evaluated on short lead-times. For longer leadtimes, the latter policy slightly under-achieves the target. Finally, for CSL $=0.95$, both policies are found to be 'unable' to meet the target. Overall, the average cost of the $\left(r_{k}, Q\right)$ is lower than that of the static policy, whereas the CSL is slightly higher in the latter case.

Individualised attention was given to each of the SKUs considered in our experiment; the dynamic policy is consistently 'good' for each individual scenario whereas the performance of the static policy is highly variable. Consider for example a demand pattern characterised by a negative trend. In this case, the static re-order point set for that policy at the end of the $N_{l}$ period will always exceed the true requirements, resulting in CSLs that exceed the targets. Correspondingly, should a positive trend be present in the data, the opposite will be the case. Similar comments can be made in the case of step-changes (up or down) in the underlying demand structures. Since results are reported across all SKUs, considerable differences cancel out, favouring the performance of the static policy. On the other hand, as discussed above, the performance of the dynamic policy is consistently 'good' for each single SKU. 
At this point we must remark that the performance of the dynamic policy is dependent upon the quality of the forecasts produced. High forecast uncertainty during the within-sample block of time and/or poor forecasts for the out-of-sample period render the performance of the $(r, Q)$ policy marginally better than that of the $\left(r_{k}, Q\right)$ policy in terms of achieved service level, whereas the cost-related results still favour the dynamic policy. However, it is natural to assume that if better forecasts had been provided (resulting in a smaller forecast uncertainty), the $\left(r_{k}, Q\right)$ policy would have out-weighted the $(r, Q)$ policy in terms of both costs and service level. This is what Babai and Dallery (2007) have shown, using theoretically generated data for one SKU.

The simulation results for the case of the relative forecast uncertainty are given in Appendix $C$. They are, overall, consistent with those reported for the absolute forecast uncertainty case. The performance of the $\left(r_{k}, Q\right)$ policy under this experimental structure is slightly worse in terms of costs, compared with the results obtained for the absolute uncertainty case.. However, the achieved service level is higher in the majority of cases.

Finally, 'few' periods in the within-sample lead to a poor representation of the PDF of the forecast uncertainty. Correspondingly, 'few' periods in the out-of-sample evaluation do not allow us to gain sufficient insight into the performance of the policies. Therefore, as expected, the empirical results show that performance of both policies is better for the scenario $N_{1}=18$ and $N_{2}=18$ periods which represents the best trade-off between the width of the within-sample and out-of-sample blocks of time.

Overall, when all scenarios are considered, and when average results are computed across all parameter combinations, there is little to choose between the two policies in terms of the CSL achieved. Nevertheless, the dynamic policy results in inventory cost reductions as high as $8.3 \%$, which constitutes a considerable difference for any realworld system. Our analysis suggests that the adoption of the dynamic policy proposed in this paper should offer tangible benefits to manufacturers dealing with the problem of setting re-order points over a long horizon. 


\section{Conclusions}

In this paper a new approach to dynamic forecast-based inventory control has been proposed. The approach has three merits: i) it is generic, since the determination of the control parameters does not depend upon any underlying demand structure and thus a corresponding 'optimal' forecast procedure; ii) it takes lead-time uncertainty into account; and iii) it is not computationally intensive compared with other dynamic approaches proposed in the literature. In particular, implementation of our approach implies that the policy parameters can be easily computed using Excel (or any other spreadsheet package). As such, we feel that its practical value is considerable since it can be easily integrated into a given inventory management solution.

Following this approach, a periodic dynamic re-order point control policy was developed, referred to as the $\left(r_{k}, Q\right)$ policy. The parameters of this policy are provided under both demand and lead-time uncertainty (variability) and for a cycle service level constraint. We showed that the re-order point $r_{k}$ can be computed numerically by using, for example, a fixed point algorithm. Results are provided for both an absolute and a relative forecast uncertainty model.

We then conducted an empirical investigation to compare the performance of the dynamic $\left(r_{k}, Q\right)$ policy (relying upon advance demand information in terms of forecasts) with the static $(r, Q)$ policy often employed in practical applications. Results have been generated for 135 SKUs from the pharmaceutical industry, under a wide range of experimental conditions. The results indicate a similar performance of the two policies in terms of service level achieved. However, the considerable inventory cost reductions obtained from the dynamic policy render its application preferable in any corresponding real-world application.

An interesting line of further research would be to consider more elaborate models for representing the forecast uncertainty, such as the mixed model, as well as the possibility of more regularly updating the demand information available. One might also extend the analysis provided in this paper to other inventory control policies such as order-up-to-level. This in fact constitutes the next step of our research. 


\section{Appendix A. Parameters of the PDF of the Cumulative Forecast Uncertainty}

The method described in this Appendix can be used to compute the parameters of the probability distribution function of the cumulative forecast uncertainty over any interval $R$. Consider a history of demands and forecasts composed of $N$ periods $(R<$ $N$ ), denoted respectively by:

$D_{j}:$ the demand at a period $j$,

$F_{i-1, j}$ : the forecast given at the end of period $i-1$ for a period $j(j \geq i)$.

We consider two models in order to represent the forecast uncertainty: an 'absolute' model and a 'relative' one. We compute, for each period $i$ in the demand history $(1 \leq i$ $\leq N-R+1)$, the value of the cumulative forecast uncertainty over the forward interval $R$, which we denote by $C F U_{i-1, R}$ as follows ${ }^{3}$ :

$$
C F U_{i-1, R}= \begin{cases}\sum_{j=i}^{i+R-1} D_{j}-\sum_{j=i}^{i+R-1} F_{i-1, j} & \text { If the forecast uncertainty is absolute } \\ \frac{\sum_{j=i}^{i+R-1} D_{j}-\sum_{j=i}^{i+R-1} F_{i-1, j}}{\sum_{j=i}^{i+R-1} F_{i-1, j}} & \text { If the forecast uncertainty is relative }\end{cases}
$$

Using $C F U_{i-1, R}$, we compute the mean and standard deviation of the cumulative forecast uncertainty over the interval $R$, denoted by $\mu_{C F U_{R}}$ and $\sigma_{C F U_{R}}$ respectively, as follows:

$\mu_{C F U_{R}}=\frac{\sum_{i=1}^{N-R+1} C F U_{i-1, R}}{N-R+1}$ and $\sigma_{C F U_{R}}=\sqrt{\frac{1}{N-R} \sum_{i=1}^{N-R+1}\left(C F U_{i-1, R}-\mu_{C F U_{R}}\right)^{2}}$

Note that in the case of the relative model, the mean and standard deviation of the cumulative forecast uncertainty are given in percentages. Thus, in order to compute the actual cumulative forecast uncertainty, the value given by the distribution has to be multiplied by the cumulative forecast over the same interval $R$.

\footnotetext{
${ }^{3}$ The end of period 0 (i.e. $i=0$ ) corresponds to the beginning of the demand history, so that $F_{0,1}$ is the forecast made at the beginning of the history for the period $i=1$.
} 


\section{Appendix B. Proof of Proposition 1}

The re-order point $r_{k}$ may be derived based on:

$P\left(\right.$ Cumulative forecasted demand over $\left.L+1 \leq r_{k}\right)=C S L$

Since the lead time $L$ is a discrete random variable, and by considering the Total Probability Theorem and the equation above, we have:

$\sum_{i} P\left(L=L_{i}\right) P\left(\right.$ Cumulative forecasted demand over $\left.L_{i}+1=r_{k}\right)=C S L$

We denote by $C F D_{k-1, L_{i}+1}$ the sum of the cumulative forecasts plus cumulative forecast uncertainty over $L_{i}+1$ periods estimated at the end of period $k-1$, and by $\Phi_{C F D_{k-1, L_{i}+1}}$ (.) its cumulative probability distribution function. At the end of each period $k-1$ :

$C F D_{k-1, L_{i}+1}=C F U_{L_{i}+1}+\sum_{j=1}^{L_{i}+1} F_{k-1, k+j-1}$

The random variable $C F D_{k-1, L_{i}+1}$ is distributed with a mean and a standard deviation, denoted by $\mu_{C F D_{k-1, L_{i}+1}}$ and $\sigma_{C F D_{k-1, L_{i}+1}}$ respectively. They are given by:

$\mu_{C F D_{k-1, L_{i}+1}}= \begin{cases}\sum_{j=1}^{L_{i}+1} F_{k-1, k+j-1}+\mu_{C F U_{L_{i}+1}} & \text { If the forecast uncertainty is absolute } \\ \sum_{j=1}^{L_{i}+1} F_{k-1, k+j-1}\left[1+\mu_{C F U_{L_{i}+1}}\right] & \text { If the forecast uncertainty is relative }\end{cases}$ and

$$
\sigma_{C F D_{k-1, L_{i}+1}}= \begin{cases}\sigma_{C F U_{L_{i}+1}} & \text { If the forecast uncertaint y is absolute } \\ \sigma_{C F U_{L_{i}+1}} \sum_{j=1}^{L_{i}+1} F_{k-1, k+j-1} & \text { If the forecast uncertainty is relative }\end{cases}
$$

The re-order point $r_{k}$ is then given by:

$\sum_{i} P\left(L=L_{i}\right) \Phi_{C F D_{k-1, L_{i}+1}}\left(r_{k}\right)=C S L$

which completes the proof of Proposition 1. 


\section{Appendix C. Simulation Results for a Relative Forecast Uncertainty}

$\ll<<$ Please insert Table 6 about here $\gg>>$

$\ll<$ Please insert Table 7 about here $\gg \gg>$

$\ll<$ Please insert Table 8 about here $\gg>$ 


\section{References}

Aggarwal, A. and Park, J. K. (1993). Improved algorithms for economic lot size problems. Operations Research, 41:549-571.

Armstrong, J. S. (2001). "Principles of Forecasting: A Handbook for Researchers and Practitioners", J. S. Armstrong, ed., Kluwer Academic Publishers, Norwell, MA.

Babaï, M. Z. and Dallery, Y. (2007). Dynamic versus static control policies in single stage production-inventory systems. Working paper, Laboratoire Génie Industriel, Ecole Centrale Paris.

Chen, F., Drezner, Z., Ryan, J. K., and Simchi-Levi, D. (2000). Quantifying the bullwhip effect in a simple supply chain: The impact of forecasting, lead-times, and information. Management Science, 46:436-443.

Ehrhardt, R. (1984). (s, S) policies for a dynamic inventory model with stochastic lead-times. Management Science, 32:121-132.

Eppen, G. D. and Martin, R. K. (1988). Determining safety stock in the presence of stochastic lead time and demand. Management Science, 34:1380-1390.

Graves, S. C. (1999). A single-item inventory model for a nonstationary demand process. Manufacturing and Service Operations Management, 1:50-61.

Hadley, G. and Whitin, T. (1963). Analysis of Inventory Systems. Prentice-Hall, NJ.

Heath, D. C. and Jackson, P. L. (1994). Modeling the evolution of demand forecasts with application to safety-stock analysis in production/distribution systems. IIE Transactions, 26:17-30.

Johnson, A. J. and Montgomery, D.C. (1974). Operations research in production planning, scheduling, and inventory control. John Wiley \& Sons, New York, USA.

Kaplan, R. (1970). A dynamic inventory model with stochastic lead-times. Management Science, 16:491-507.

Karlin, S. (1960). Dynamic inventory policy with varying stochastic demands. Management Science, 6:231-258.

Lee, H. L., Padmanabhan, P., and Whang, S. (1997). Information distortion in a supply chain: The bullwhip effect. Management Science, 43:546-558.

Nevison, C. and Burstein, M. (1984). The dynamic lot-size model with stochastic lead-times. Management Science, 30:100-109.

Scarf, H. (1959). The optimality of $(\mathrm{s}, \mathrm{S})$ policies in the dynamic inventory problem. Mathematical Methods in the Social Sciences, Stanford University Press, Stanford, California. 
Silver, E. A. (1978). Inventory control under a probabilistic time-varying, demand pattern. AIIE Transactions, pages 371-379.

Speh, T. W. and Wagenheim, G. (1978). Demand and lead-time uncertainty: the impacts of physical distribution performance and management. Journal of Business Logistics, 1:95-113.

Strijbosch, L., Heuts, R., and van der Schoot E.H.M. (2000). A combined forecast-inventory control procedure for spare parts. Journal of the Operational Research Society, 51:1184-1192.

Syntetos, A. and Boylan, J. E. (2006). On the stock control performance of intermittent demand estimators. International Journal of Production Economics, 103:36-47.

Syntetos, A., Nikolopoulos, K., Boylan, J. E., Fildes, R., and Goodwin, P. (2007). The effects of integrating management judgement into intermittent demand forecasts. International Journal of Production Economics, forthcoming.

Tang, O. and Grubbström, R. W. (2004). Considering stochastic lead-times in a manufacturing/remanufacturing system with deterministic demands and return. International Journal of Production Economics, 93-94:285-300.

Wagelmans, A. P. M., Van Hoesel, C. P. M., and Kolen, A. W. J. (1992). Economic lotsizing: an $\mathrm{O}(\mathrm{n} \log \mathrm{n})$ algorithm that runs in linear time in the Wagner-Whitin case. Operations Research 40:145-156.

Wagner, H. M. and Whitin, T. M. (1958). Dynamic version of the economic lot size model. Management Science, 5:89-96.

Whybark, D. C and Williams, J. G. (1976). Material requirements planning under uncertainty. Decision Science, 7:595-606.

Zipkin, P. H. (2000). Foundations of Inventory Management. McGraw-Hill, USA. 


\section{List of figures}

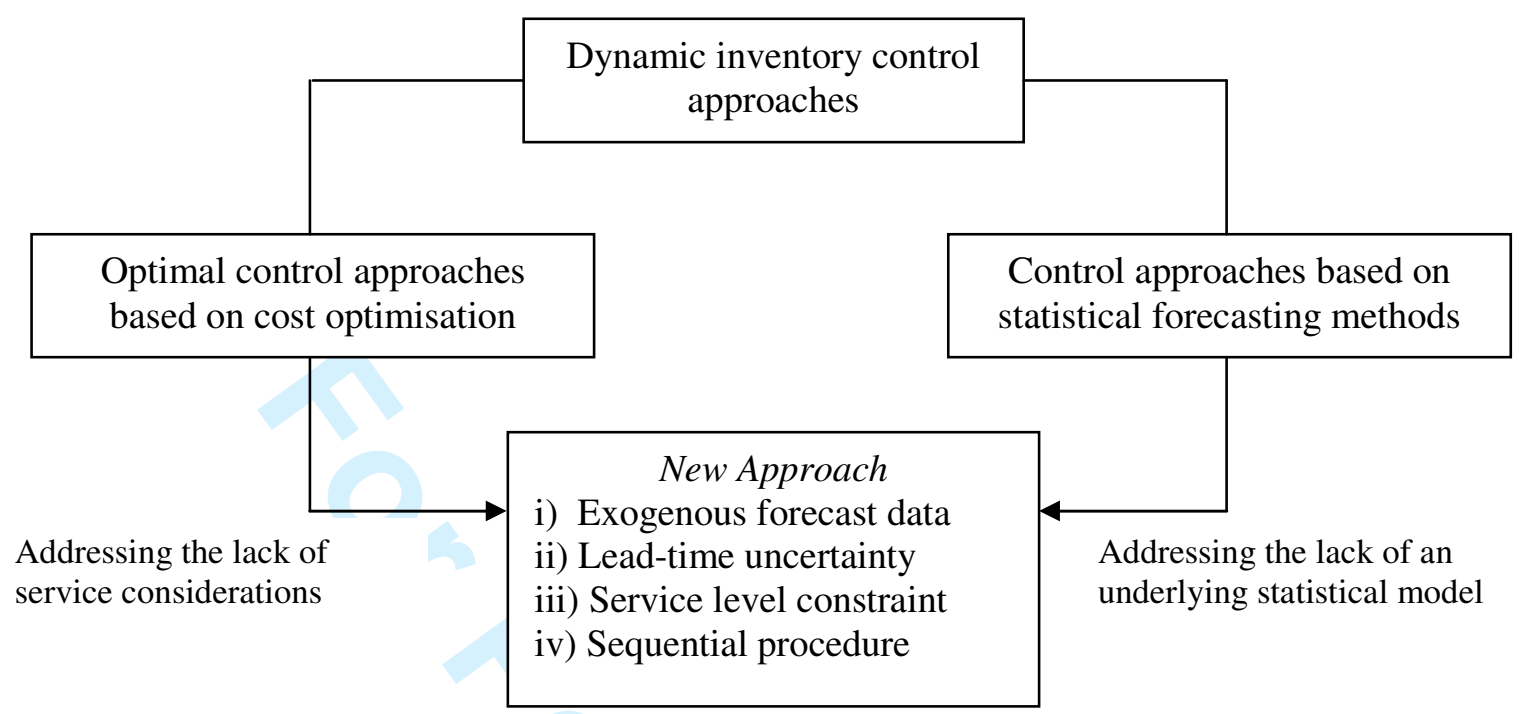

Figure 1. A new dynamic control approach

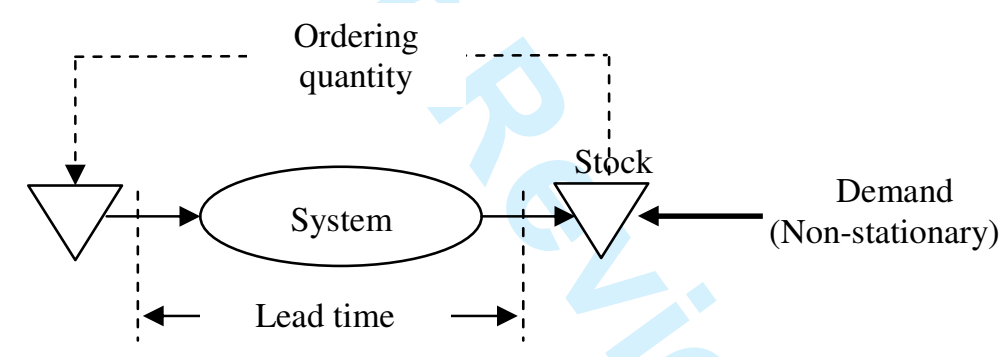

Figure 2. The inventory system model

Forecasts and uncertainties

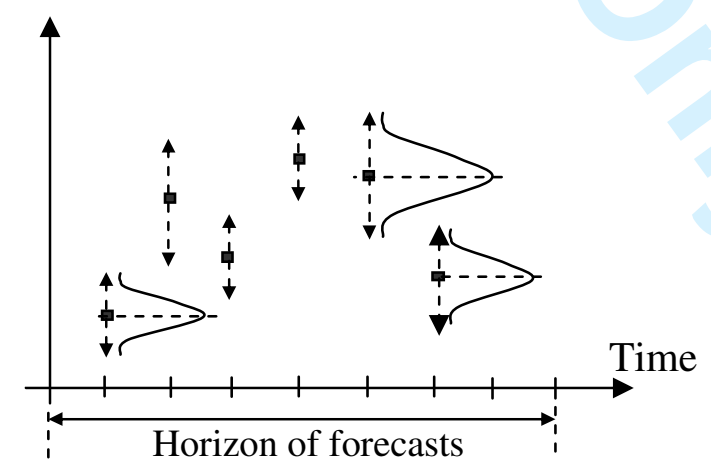

Figure 3. The demand model 


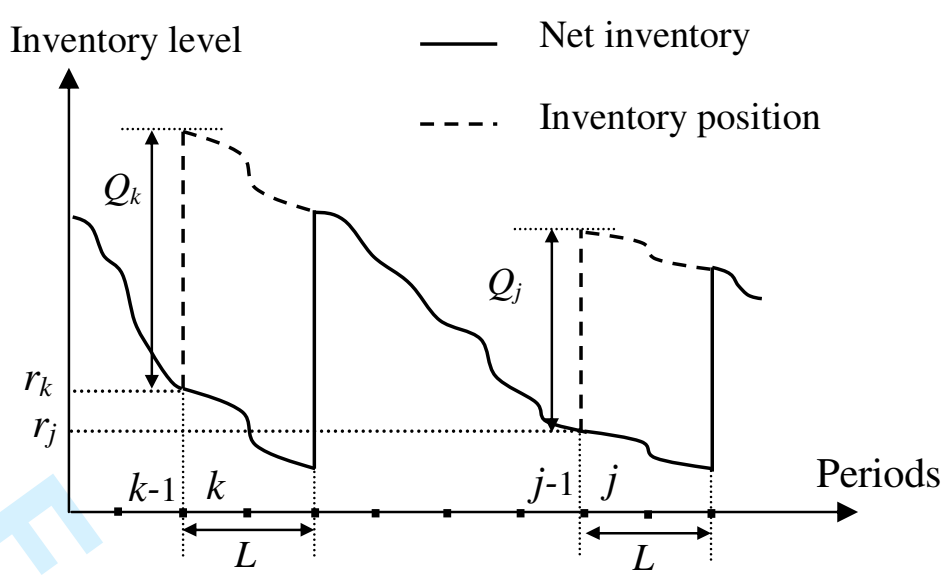

Figure 4. The $\left(r_{k}, Q\right)$ policy

\begin{tabular}{|c|c|}
\hline Within-Sample & Out-Of-Sample \\
\hline Width $\left(N_{1}\right): 15,18,21$ periods & Width $\left(N_{2}\right): 36-N_{1}$ periods \\
\hline Forecast Uncertainty: Absolute, Relative & Lead Time: $\mu_{L}=1,2,3$ periods \\
\hline & Target $\mathrm{CSL}=0.8,0.85,0.9,0.95$ \\
\hline
\end{tabular}

Figure 5. Simulation scenarios 


\begin{tabular}{|l|l|c|c|c|c|c|c|c|c|}
\hline \multirow{2}{*}{$\begin{array}{l}\text { Stock control } \\
\text { results across } \\
\text { all 135 SKUs }\end{array}$} & \multicolumn{6}{|c|}{ Within-Sample =21 periods / Out-Of-Sample = 15 periods } \\
\cline { 2 - 10 } & $(r, Q)$ & $\left(r_{k}, Q\right)$ & $(r, Q)$ & $\left(r_{k}, Q\right)$ & $(r, Q)$ & $\left(r_{k}, Q\right)$ & \multicolumn{2}{c|}{$C S L, Q)$} & $\left(r_{k}, Q\right)$ \\
\hline \multirow{2}{*}{$\mu_{L}=3$} & Cost & 72012.51 & 69039.08 & 72433.09 & 69364.64 & 72796.03 & 69549.09 & 73349.05 & 69993.77 \\
\cline { 2 - 10 } & $S L$ & 0.882 & 0.860 & 0.891 & 0.869 & 0.909 & 0.889 & 0.927 & 0.908 \\
\hline \multirow{2}{*}{$\mu_{L}=2$} & Cost & 66834.21 & 63922.25 & 67015.99 & 64112.31 & 67105.52 & 64126.22 & 69133.11 & 64213.24 \\
\cline { 2 - 10 } & $S L$ & 0.884 & 0.865 & 0.898 & 0.882 & 0.912 & 0.902 & 0.931 & 0.920 \\
\hline \multirow{2}{*}{$\mu_{L}=1$} & Cost & 63275.04 & 60007.35 & 63305.58 & 60131.41 & 63616.42 & 60470.75 & 65979.29 & 61066.15 \\
\cline { 2 - 10 } & $S L$ & 0.899 & 0.884 & 0.906 & 0.904 & 0.923 & 0.919 & 0.937 & 0.932 \\
\hline
\end{tabular}

Table 3. Empirical results for $N_{1}=21 / N_{2}=15$

\begin{tabular}{|l|l|c|c|c|c|c|c|c|c|}
\hline \multirow{2}{*}{$\begin{array}{l}\text { Stock control } \\
\text { results across } \\
\text { all 135 SKUs }\end{array}$} & \multicolumn{6}{|c|}{ Within-Sample $=18$ periods / Out-Of-Sample $=18$ periods } \\
\cline { 2 - 10 } & $(r, Q)$ & $\left(r_{k}, Q\right)$ & $(r, Q)$ & $\left(r_{k}, Q\right)$ & $(r, Q)$ & $\left(r_{k}, Q\right)$ & \multicolumn{2}{c|}{$C S L, Q)$} & $\left(r_{k}, Q\right)$ \\
\hline \multirow{2}{*}{$\mu_{L}=3$} & Cost & 69639.51 & 66099.07 & 70110.30 & 66246.50 & 71111.18 & 66874.04 & 73052.52 & 67024.82 \\
\cline { 2 - 10 } & $S L$ & 0.882 & 0.869 & 0.891 & 0.870 & 0.911 & 0.891 & 0.927 & 0.909 \\
\hline \multirow{2}{*}{$\mu_{L}=2$} & Cost & 64147.04 & 62575.88 & 64696.40 & 62793.40 & 65554.37 & 62994.08 & 67965.90 & 63050.72 \\
\cline { 2 - 10 } & $S L$ & 0.892 & 0.869 & 0.904 & 0.887 & 0.916 & 0.903 & 0.933 & 0.922 \\
\hline \multirow{2}{*}{$\mu_{L}=1$} & Cost & 61304.38 & 59078.31 & 61401.35 & 59078.31 & 61895.51 & 59489.99 & 63645.84 & 59896.49 \\
\cline { 2 - 10 } & $S L$ & 0.899 & 0.884 & 0.911 & 0.905 & 0.923 & 0.920 & 0.939 & 0.933 \\
\hline
\end{tabular}

Table 4. Empirical results for $N_{1}=18 / N_{2}=18$ 


\begin{tabular}{|l|l|c|c|c|c|c|c|c|c|}
\hline \multirow{2}{*}{$\begin{array}{l}\text { Stock control } \\
\text { results across } \\
\text { all 135 SKUs }\end{array}$} & \multicolumn{8}{|c|}{ Within-Sample $=21$ periods / Out-Of-Sample $=15$ periods } \\
\cline { 2 - 10 } & $(r, Q)$ & $\left(r_{k}, Q\right)$ & $(r, Q)$ & $\left(r_{k}, Q\right)$ & $(r, Q)$ & $\left(r_{k}, Q\right)$ & \multicolumn{2}{c|}{$C S L, Q)$} & $\left(r_{k}, Q\right)$ \\
\hline \multirow{2}{*}{$\mu_{L}=3$} & Cost & 72012.51 & 70534.77 & 72433.09 & 70747.81 & 72796.03 & 70952.70 & 73349.05 & 71137.62 \\
\cline { 2 - 10 } & $S L$ & 0.882 & 0.858 & 0.891 & 0.874 & 0.909 & 0.894 & 0.927 & 0.908 \\
\hline \multirow{2}{*}{$\mu_{L}=2$} & Cost & 66834.21 & 66318.31 & 67015.99 & 66389.13 & 67105.52 & 67078.6 & 69133.11 & 67154.56 \\
\cline { 2 - 10 } & $S L$ & 0.884 & 0.873 & 0.898 & 0.887 & 0.912 & 0.904 & 0.931 & 0.924 \\
\hline \multirow{2}{*}{$\mu_{L}=1$} & Cost & 63275.04 & 63270.25 & 63305.58 & 63310.69 & 63616.42 & 63592.165 & 65979.29 & 64328.13 \\
\cline { 2 - 10 } & $S L$ & 0.899 & 0.887 & 0.906 & 0.903 & 0.923 & 0.920 & 0.937 & 0.932 \\
\hline
\end{tabular}

Table 6. Empirical results for $N_{1}=21 / N_{2}=15$ (Relative Forecast Uncertainty)

\begin{tabular}{|l|l|c|c|c|c|c|c|c|c|}
\hline \multirow{2}{*}{$\begin{array}{l}\text { Stock control } \\
\text { results across } \\
\text { all 135 SKUs }\end{array}$} & \multicolumn{6}{|c|}{ Within-Sample $=18$ periods / Out-Of-Sample $=18$ periods } \\
\cline { 2 - 10 } & $(r, Q)$ & $\left(r_{k}, Q\right)$ & $(r, Q)$ & $\left(r_{k}, Q\right)$ & $(r, Q)$ & $\left(r_{k}, Q\right)$ & \multicolumn{2}{c|}{$C S L, Q)$} & $\left(r_{k}, Q\right)$ \\
\hline \multirow{2}{*}{$\mu_{L}=3$} & Cost & 69639.51 & 67105.28 & 70110.30 & 67511.52 & 71111.18 & 67954.17 & 73052.52 & 69103.53 \\
\cline { 2 - 10 } & $S L$ & 0.882 & 0.858 & 0.891 & 0.879 & 0.911 & 0.894 & 0.927 & 0.909 \\
\hline \multirow{2}{*}{$\mu_{L}=2$} & Cost & 64147.04 & 64001.89 & 64696.40 & 64654.22 & 65554.37 & 65318.58 & 67965.90 & 66171.56 \\
\cline { 2 - 10 } & $S L$ & 0.892 & 0.877 & 0.904 & 0.892 & 0.916 & 0.907 & 0.933 & 0.924 \\
\hline \multirow{2}{*}{$\mu_{L}=1$} & Cost & 61304.38 & 61118.66 & 61401.35 & 61303.90 & 61895.51 & 61423.65 & 63645.84 & 62923.28 \\
\cline { 2 - 9 } & $S L$ & 0.899 & 0.890 & 0.911 & 0.906 & 0.923 & 0.921 & 0.939 & 0.935 \\
\hline
\end{tabular}

Table 7. Empirical results for $N_{1}=18 / N_{2}=18$ (Relative Forecast Uncertainty)

\begin{tabular}{|l|l|c|c|c|c|c|c|c|c|}
\hline \multirow{2}{*}{$\begin{array}{l}\text { Stock control } \\
\text { results across } \\
\text { all 135 SKUs }\end{array}$} & \multicolumn{6}{|c|}{ Within-Sample $=15$ periods / Out-Of-Sample $=21$ periods } \\
\cline { 2 - 10 } & $(r, Q)$ & $\left(r_{k}, Q\right)$ & $(r, Q)$ & $\left(r_{k}, Q\right)$ & $(r, Q)$ & $\left(r_{k}, Q\right)$ & \multicolumn{2}{c|}{$C S L=0.95$} \\
\hline \multirow{2}{*}{$\mu_{L}=3$} & Cost & 68204.81 & 67045.04 & 69022.35 & 67649.46 & 70164.15 & 68294.50 & 71629.93 & 69484.50 \\
\cline { 2 - 10 } & $S L$ & 0.874 & 0.859 & 0.889 & 0.872 & 0.906 & 0.890 & 0.924 & 0.908 \\
\hline \multirow{2}{*}{$\mu_{L}=2$} & Cost & 64076.61 & 64096.74 & 64861.05 & 64466.32 & 65266.22 & 64723.67 & 67636.55 & 67634.86 \\
\cline { 2 - 10 } & $S L$ & 0.884 & 0.877 & 0.901 & 0.890 & 0.912 & 0.907 & 0.929 & 0.918 \\
\hline \multirow{2}{*}{$\mu_{L}=1$} & Cost & 60306.88 & 61103.26 & 60514.40 & 61312.52 & 61814.88 & 61634.67 & 63731.51 & 62962.38 \\
\cline { 2 - 10 } & $S L$ & 0.899 & 0.886 & 0.906 & 0.903 & 0.923 & 0.917 & 0.937 & 0.932 \\
\hline
\end{tabular}

Table 8. Empirical results for $N_{1}=15 / N_{2}=21$ (Relative Forecast Uncertainty) 